\title{
Direct comparison of the histidine-rich protein-2 enzyme-linked immunosorbent assay (HRP-2 ELISA) and malaria SYBR green I fluorescence (MSF) drug sensitivity tests in Plasmodium falciparum reference clones and fresh ex vivo field isolates from Cambodia
}

\author{
Suwanna Chaorattanakawee', Stuart D Tyner', Chanthap Lon', Kritsanai Yingyuen', Wiriya Ruttvisutinunt', \\ Siratchana Sundrakes', Piyaporn Sai-gnam', Jacob D Johnson², Douglas S Walsh', David L Saunders ${ }^{1}$ \\ and Charlotte A Lanteri ${ }^{*}$
}

\begin{abstract}
Background: Performance of the histidine-rich protein-2 enzyme-linked immunosorbent assay (HRP-2 ELISA) and malaria SYBR Green I fluorescence (MSF) drug sensitivity tests were directly compared using Plasmodium falciparum reference strains and fresh ex vivo isolates from Cambodia against a panel of standard anti-malarials. The objective was to determine which of these two common assays is more appropriate for studying drug susceptibility of "immediate ex vivo" (IEV) isolates, analysed without culture adaption, in a region of relatively low malaria transmission.

Methods: Using the HRP-2 and MSF methods, the 50\% inhibitory concentration $\left(I C_{50}\right)$ values against a panel of malaria drugs were determined for $P$. falciparum reference clones (W2, D6, 3D7 and K1) and 41 IEV clinical isolates from an area of multidrug resistance in Cambodia. Comparison of the $I_{50}$ values from the two methods was made using Wilcoxon matched pair tests and Pearson's correlation. The lower limit of parasitaemia detection for both methods was determined for reference clones and IEV isolates. Since human white blood cell (WBC) DNA in clinical samples is known to reduce MSF assay sensitivity, SYBR Green I fluorescence linearity of $P$. falciparum samples spiked with WBCs was evaluated to assess the relative degree to which MSF sensitivity is reduced in clinical samples.

Results: $I C_{50}$ values correlated well between the HRP-2 and MSF methods when testing either P. falciparum reference clones or IEV isolates against 4-aminoquinolines (chloroquine, piperaquine and quinine) and the quinoline methanol mefloquine (Pearson $r=0.85-0.99$ for reference clones and 0.56-0.84 for IEV isolates), whereas a weaker $I C_{50}$ value correlation between methods was noted when testing artemisinins against reference clones and lack of correlation when testing IEV isolates. The HRP-2 ELISA produced a higher overall success rate $\left(90 \%\right.$ for producing $I C_{50}$ best-fit sigmoidal curves), relative to only a 40\% success rate for the MSF assay, when evaluating ex vivo Cambodian isolates. Reduced sensitivity of the MSF assay is likely due to an interference of WBCs in clinical samples.

(Continued on next page)
\end{abstract}

\footnotetext{
*Correspondence: charlottelanteri@afrims.org

'Department of Immunology and Medicine, Armed Forces Research Institute of Medical Sciences, Bangkok, Thailand

Full list of author information is available at the end of the article
} 
(Continued from previous page)

Conclusions: For clinical samples not depleted of WBCs, HRP-2 ELISA is superior to the MSF assay at evaluating fresh $P$. falciparum field isolates with low parasitaemia (<0.2\%) generally observed in Southeast Asia.

Keywords: Immediate ex vivo Plasmodium falciparum drug susceptibility testing, HRP-2 ELISA, Malaria SYBR green fluorescence assay, Cambodia

\section{Background}

In vitro testing of Plasmodium falciparum field isolates for susceptibility against currently applied anti-malarials provides an early warning of drug failure and possible clinical resistance. Several methods are commonly used to measure $P$. falciparum in vitro drug susceptibility, such as the schizont maturation test $[1],\left[{ }^{3} \mathrm{H}\right]$-hypoxanthine incorporation [2], histidine-rich protein-2 enzyme linked immunosorbent assay (HRP-2 ELISA) [3,4], and most recently the malaria SYBR Green I fluorescence (MSF) assay $[5,6]$. The schizont maturation assay is based on microscopic examination for P. falciparum blood stage growth during drug exposure. Although relatively inexpensive, this assay is labour intensive and interpretation of results is subjective. The $\left[{ }^{3} \mathrm{H}\right]$-hypoxanthine uptake assay involves measuring parasite growth by recording levels of hypoxanthine incorporated into parasite DNA [2]. While this method provides accurate and reliable results, the major drawback is the use of radioactivity, the safe disposal of which demands substantial resources. The HRP-2 method assesses parasite growth by using colorimetric ELISA to measure P. falciparum HRP-2 protein. This method offers a solution to examining drug susceptibilities of field isolates using a non-radioactive procedure that is also more cost effective compared to the $\left[{ }^{3} \mathrm{H}\right]$-hypoxanthine method [3]. Recently, the MSF assay was developed based on measuring the incorporation of the fluorescent SYBR Green I dye into parasite DNA. This method relies on a single step of DNA staining, which is less labour intensive compared to an ELISA method, and is more amenable for high throughput screening of new drug candidates [7]. However, utility of the MSF assay in clinical isolates could be compromised in samples with a relatively low parasitaemia, because of non-specific fluorescence background attributed to SYBR Green I binding to human DNA of white blood cells (WBCs) that may reduce sensitivity of parasite detection [8].

Since 2004, Armed Forces Research Institute of Medical Sciences (AFRIMS) routinely applies the HRP-2 ELISA for "immediate ex vivo" (IEV) drug susceptibility testing of fresh P. falciparum field isolates without culture adaptation from multidrug resistant areas in Cambodia and Thailand [9-12]. This method generates $\mathrm{IC}_{50}$ results comparable to the World Health Organization (WHO) microplate schizont maturation test and the $\left[{ }^{3} \mathrm{H}\right]$-hypoxanthine uptake assay, while offering the advantages of rapidity relative to the schizont maturation test and avoiding radioisotope use [4].
Moreover, measuring drug susceptibility of fresh parasite isolates without culture adaptation provides results that more accurately reflect the overall drug susceptibility phenotype of an in vivo infection by avoiding loss of drugresistant parasite subpopulations during culture adaptation [13-15]. The HRP-2 ELISA performs robustly when analysing fresh isolates of relatively low parasitaemia. For example, in a previous related field investigation of malaria drug resistance, the HRP-2 assay had a success rate of $75 \%$ for $\mathrm{IC}_{50}$ value determination in samples collected during a survey in Cambodia and Thailand of 590 P. falciparum IEV isolates, of which nearly half had $<0.2 \%$ parasite density [9].

Recently, several papers evaluated the utility of the MSF assay for measuring the drug susceptibility of $P$. falciparum reference and field isolates from Africa, where malaria transmission and thus patient parasitaemia are commonly higher than in Southeast Asia. The MSF assay produces comparable $\mathrm{IC}_{50}$ results to those generated by traditional $\left[{ }^{3} \mathrm{H}\right]$-hypoxanthine incorporation and HRP-2 ELISA methods $[5,6,16,17]$, but presumably only when parasitaemia is sufficiently high to produce reliable results. For example, a study conducted in Madagascar reports that the MSF method works well, with an overall $80 \%$ success rate of generating $\mathrm{IC}_{50}$ results when testing fresh $P$. falciparum samples of least $0.1 \%$ parasitaemia and washed three times prior to plating [17]. Another report [16] using fresh isolates from non-immune travellers who acquired malaria in West Africa indicates that the MSF assay yields the same success rate as the HRP-2 ELISA method when analysing samples with a mean parasitaemia of $0.75 \%$, also washed three times prior to plating. In a recent investigation [6] of IEV isolates collected from western Kenya, the MSF assay produced a success rate of $78 \%$ with a parasitaemia $>0.5 \%$, whereas the success rate decreased to only $61 \%$ in samples with $\leq 0.5 \%$.

However, the MSF assay is not expected to perform well to evaluate field isolates in whole blood samples collected from patients with lower parasitaemia. For example, a comparison of the MSF and HRP-2 ELISA methods for measuring in vitro susceptibilities of $P$. falciparum laboratory reference strains showed that the HRP-2 assay provides a similar limit of detection in either whole blood-media mixtures or WBC-free samples, whereas sensitivity of the MSF assay diminishes greatly in samples spiked with WBCs as a result of confounding fluorescence 
background signal [8]. This confounding effect, likely a result of SYBR Green I binding to WBC DNA, is further problematic in P. falciparum reference samples of lower parasitaemia $<1.0 \%$ [8], as is typically encountered in regions of low malaria transmission. To date, a direct comparison of MSF versus HRP-2 methods has not been conducted using fresh clinical isolates of P. falciparum with such low parasitaemia.

The study described herein was conducted to evaluate utility of the HRP-2 ELISA versus MSF methods for determining ex vivo drug susceptibility of fresh $P$. falciparum isolates from Cambodia, representing an area of relatively low parasite transmission and thus low parasite density in patients. The hypothesis was that the HRP-2 ELISA would be more sensitive than the MSF assay when testing fresh clinical isolates of low parasitaemia $(<0.2 \%)$.

\section{Methods}

Plasmodium falciparum culture and synchronization

Plasmodium falciparum reference strains W2, D6, 3D7, and K1 were maintained as described previously [18]. Briefly, cryopreserved vials of reference clones were recovered using 3.5\% sodium chloride [19] and maintained in RPMI-1640 medium containing 25 mM HEPES, $25 \mathrm{mM}$ sodium bicarbonate, $5 \%$ human $\mathrm{O}^{+}$erythrocytes, $10 \%$ pooled $\mathrm{AB}^{+}$serum and $0.1 \mathrm{mg} / \mathrm{mL}$ gentamycin at $37^{\circ} \mathrm{C}$ with $5 \%$ $\mathrm{CO}_{2}, 5 \% \mathrm{O} 2$, and $90 \% \mathrm{~N}_{2}$. To obtain a predominance $(\geq 90 \%)$ of ring stage parasites for drug susceptibility testing, $5 \% \mathrm{D}$-sorbitol synchronization was performed as previously described [20,21]. After synchronization, parasites were maintained for 48 hours prior to conducting the drug susceptibility assay.

\section{Plasmodium falciparum isolate collection and sample processing}

Clinical P. falciparum isolates were obtained from patients with uncomplicated falciparum malaria enrolled in an antimalarial drug resistance surveillance study in Cambodia. The study was approved by the Cambodian National Ethics Committee for Health Research (NECHR), and the Walter Reed Army Institute of Research (WRAIR) Institutional Review Board (protocol number: WRAIR 1576). After signing informed consent, malaria patients $\geq 13$ years old without a history of anti-malarial drug use within the previous seven days were enrolled into the study. Diagnosis of malaria was conducted using Giemsa-stained peripheral blood smears, with microscopy species determination verified using real-time PCR targeting the $18 \mathrm{~s}$ rRNA as reported elsewhere [9]. A total of 41 isolates, collected from volunteers with mono $P$. falciparum infection living in northern (Preah vihear province) and southern Cambodia (Kampong Spou, Kampot, and Preah Sihanouk provinces) during May -October 2011, were evaluated in the HRP-2 and MSF assays. At the time of diagnosis (before treatment), patient blood samples (4 $\mathrm{ml}$ of venous blood in sodium heparin tubes) were tested for IEV drug susceptibility, without a leukocyte depletion step or culture adaptation, as previously described [9]. Samples were applied to dried drug plates for HRP-2 and MSF assay analysis within six hours after phlebotomy.

\section{Preparation of dried drug plates}

Dried drug plates, for use in the HRP-2 and MSF assays, were prepared using published methods [9]. Briefly, six drugs, dihydroartemisinin (DHA), artesunate (AS), mefloquine hydrochloride (MQ), quinine sulphate hydrate $(\mathrm{QN})$, chloroquine diphosphate (CQ), and anhydrous piperaquine phosphate (PPQ), were coated onto 96-well plates in duplicate. Molecular weights of DHA, AS, MQ, QN, CQ, and PPQ used were 284.35, 384.42, 414.78, 782.97, 515.92, and $927.57 \mathrm{~g} / \mathrm{mol}$, respectively. All test drugs were provided by the WRAIR (Silver Spring, MD, USA). Drugs were dissolved in $70 \%$ ethanol to make $1 \mathrm{mg} / \mathrm{ml}$ stock solutions, with the exception of using $0.5 \%$ lactic acid in distilled water as a solvent for PPQ. The drug stock solutions were then diluted to appropriate concentrations in sterile distilled water. Three-fold serial drug dilutions were performed on plates to reach final concentrations (after $200 \mu \mathrm{L}$ of sample added) ranging from: 0.027 to $20 \mathrm{ng} / \mathrm{ml}$ for DHA and AS, 0.274 to $200 \mathrm{ng} / \mathrm{ml}$ for $\mathrm{MQ}, 1.71$ to $1,250 \mathrm{ng} / \mathrm{mL}$ for $\mathrm{QN}, 2.74$ to $2,000 \mathrm{ng} / \mathrm{ml}$ for $\mathrm{CQ}$, and 0.86 to $625 \mathrm{ng} / \mathrm{mL}$ for PPQ. The top row of each plate served as a drug-free control for parasite growth. Drug plates were dried overnight in a running biosafety cabinet and stored at $4^{\circ} \mathrm{C}$ up to eight weeks prior to use. As a quality control for dried drug plate integrity during transport to and from field sites, a subset of plates not used in the assays were tested to ensure an acceptable range of $\mathrm{IC}_{50}$ values was attained against the $P$. falciparum $\mathrm{W} 2$ reference clone, as described previously [21].

\section{HRP-2 ELISA and MSF Plasmodium falciparum susceptibility testing}

Performance of HRP-2 ELISA and MSF methods was evaluated side-by-side in $P$. falciparum reference lines and in Cambodian IEV isolates. Each reference clone or fresh isolate sample was added to the same drug-coated plates, which were later processed for each assay as described below. Synchronized cultures of P. falciparum reference strains with $\geq 90 \%$ ring forms were diluted to $0.5 \%$ parasitaemia with $1.5 \%$ haematocrit in $0.5 \%$ Albumax RPMI 1640, and transferred to dried drug-coated plates. Plates were incubated at $37^{\circ} \mathrm{C}$ with $5 \% \mathrm{CO}_{2}, 5 \% \mathrm{O}_{2}$, and $90 \% \mathrm{~N}_{2}$ for 72 hours. For IEV drug susceptibility testing, blood samples with $\leq 0.5 \%$ parasitaemia were transferred to dried drug-coated plates without sample dilution and those with $>0.5 \%$ parasitaemia were diluted to $0.2-0.5 \%$ 
prior to plating. Plates containing IEV isolates were incubated at $37^{\circ} \mathrm{C}$ using a candle jar, instead of mixed gas.

After 72 hours of incubation, plates were frozen and thawed and then analysed in parallel for parasite growth inhibition by HRP-2 and MSF assay. For HRP-2 assay analysis, culture samples were diluted before performing ELISA as described in Rutvisuttinunt et al. [21] and Tyner et al. [9]. The MSF assay was performed according to Johnson et al. [5]. Briefly, for the MSF assay $100 \mu \mathrm{L}$ of samples per well were transferred to Optiplate-96 F black plates (Perkin Elmer, Salem, MA, USA) prior to adding $100 \mu \mathrm{L}$ lysis buffer [20 mM Tris (pH 7.5), 5 mM EDTA, $0.008 \%$ (wt/vol) saponin, and $0.08 \%$ (vol/vol) Triton X-100] containing SYBR Green I (1X final concentration). Plates were incubated for one hour in the dark at room temperature and then relative fluorescence unit (RFU) measurements in each well were made using a Victor plate reader (Perkin Elmer, Salem, MA, USA) at an excitation/ emission wavelength of 485/535 nm. HRP-2 optical densities (OD) and SYBR Green RFU of duplicate wells were averaged and plotted against drug concentrations. $\mathrm{IC}_{50}$ values were estimated by nonlinear regression analysis using the ICEstimator program [22].

\section{Assessing SYBR Green fluorescence linearity and detection limit for parasitaemia}

SYBR Green fluorescence linearity of parasitaemia between $0-10 \%$ was examined by serially diluting synchronized P. falciparum ring forms of strains 3D7 and K1 with non-infected erythrocytes as described previously [5]. Briefly, two-fold serial dilutions of parasite cultures were performed using non-infected erythrocytes with $1.5 \%$ haematocrit in culture medium. Then $100 \mu \mathrm{L}$ cell media mixture with varying levels of parasitaemia and non-infected erythrocyte samples were added in 12 replicate wells in each row of the 96-well plates. Next, $100 \mu \mathrm{L}$ lysis buffer containing SYBR Green I dye (1X final concentration) was added before incubating plates for one hour in the dark at room temperature, followed by taking RFU measurements as previously described. To assess fluorescence linearity in $P$. falciparum samples spiked with WBCs, similar procedures were done, but using only $50 \mu \mathrm{L}$ of samples with varying parasite densities and noninfected erythrocyte samples in 12 replicate wells of each row. Next $50 \mu \mathrm{L}$ of $1.5 \%$ haematocrit blood containing $17,000 \mathrm{WBC} / \mu \mathrm{L}$ was added to yield samples with $0-5 \%$ parasitaemia containing $8,500 \mathrm{WBC} / \mu \mathrm{L}$, representing maximum WBC counts observed in malaria patients in Cambodia [10]. After subtraction of non-infected erythrocyte background, average RFUs of 12 replicate wells were plotted on the $\mathrm{Y}$ axis against \% parasitaemia on the $\mathrm{X}$ axis. Linear regression line, $\mathrm{r}^{2}$, limit of detection (LOD), and limit of quantitation (LOQ) were determined using MS Excel. Up to three experiments were conducted to yield the LOD and LOQ range, calculated as per International Conference on Harmonization ( $\mathrm{ICH}$ ) guidelines using the equations below.

$$
\begin{aligned}
L O D= & 3 * \text { the residual standard deviation } \\
& \quad / \text { slope of a regression line } \\
L O Q= & 10 * \text { LOD } / 3
\end{aligned}
$$

\section{Reliability of HRP-2 and MSF drug susceptibility assays in} samples with low parasitaemia and effect of \% parasitaemia on $\mathrm{IC}_{50}$ values

To examine performance of HRP-2 ELISA and MSF drug susceptibility methods in samples with low parasitaemia, $P$. falciparum W2 and D6 parasites were applied to dried drug plates at varying parasitaemia of $0.005,0.01,0.05$, 0.2 , and $0.5 \%$ and the assays were conducted as described above. Two experiments were performed for each reference strain. The lowest tested parasitaemia that produced best-fit sigmoidal concentration-response curves resulting in $\mathrm{IC}_{50}$ values was determined for each method. In addition, $\mathrm{IC}_{50}$ values obtained from varying parasitaemia were compared to investigate the effect of baseline parasitaemia (inoculum effect) on $\mathrm{IC}_{50}$ values.

For IEV drug susceptibility testing, reliability of the HRP-2 and MSF methods in samples with low parasitaemia was evaluated as the assay success rate when testing DHA, AS, MQ, QN, CQ, and PPQ activities. A "successful" $\mathrm{IC}_{50}$ assay result for each $P$. falciparum clinical isolate was defined as achieving a sigmoidal concentration-response with an $\mathrm{IC}_{50}$ confidence interval ratio $\leq 5$ when testing six serial drug dilutions for at least one of the tested drugs.

\section{Statistical analysis}

$\mathrm{IC}_{50}$ values obtained from HRP-2 ELISA and MSF methods were compared using Wilcoxon matched pair tests. Log transformed $\mathrm{IC}_{50}$ values were utilized to analyse the correlation and agreement between the two methods using Pearson's correlation and Bland-Altman plots, respectively $[4,23]$. The Kruskal-Wallis test was performed to examine the effect of baseline parasitaemia on HRP-2 ELISA IC $_{50}$ values. Statistical analysis was performed using GraphPad Prism (GraphPad Software, Inc, San Diego, CA, USA).

\section{Results}

\section{SYBR green fluorescence linearity}

The linearity of SYBR Green fluorescence in P. falciparum reference strain samples was compared with and without the addition of WBCs (Figure 1). For samples without WBCs, a range of MSF assay LOD and LOQ values of $0.13-0.15 \%$ and $0.43-0.50 \%$ parasitaemia, respectively, was determined from the results of three independent experiments using either $P$. falciparum 3D7 or K1 reference clones. In contrast, the MSF assay appears to lose 


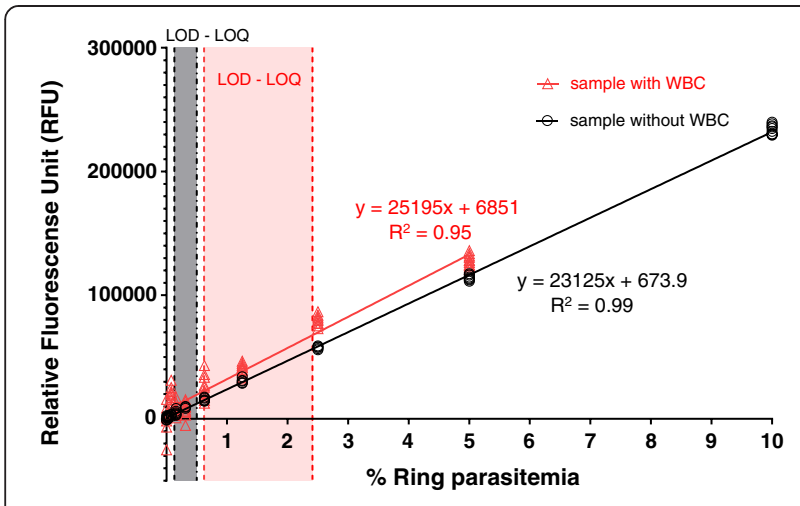

Figure 1 SYBR Green I fluorescence linearity in Plasmodium falciparum ring stages of $0-10 \%$ parasitaemia. Data points show average RFUs of 12 replicate wells, derived from one experiment using the $P$. falciparum $\mathrm{K} 1$ clone, plotted on the $Y$ axis against \% parasitaemia on the $X$ axis. Red and black symbols, lines, and letters represent samples spiked with $8,500 \mathrm{WBC} / \mu \mathrm{L}$ and samples without WBCs, respectively. Regression line and $R^{2}$ are shown. Dashed lines indicate $L O D$ and $L O Q$ range parasitaemia values obtained from up to three experiments.

sensitivity in samples spiked with WBCs, as evidenced by higher LOD and LOQ values of $0.62 / 0.72 \%$ and $2.06 /$ $2.41 \%$ parasitaemia, respectively, from two independent experiments.

\section{HRP-2 and MSF assay comparisons In vitro drug susceptibility testing}

The HRP-2 and MSF assays were evaluated simultaneously using a $0.5 \%$ baseline parasitaemia of in vitro P. falciparum reference strains with varying drug susceptibility profiles: W2 and K1 (susceptible to mefloquine, and resistant to chloroquine), D6 (susceptible to chloroquine, and resistant to mefloquine), and 3D7 (susceptible to chloroquine, but less susceptible to mefloquine than W2). From the $\mathrm{IC}_{50}$ profiles of all parasite laboratory strains (Figure 2A), both HRP-2 and MSF assays consistently differentiated sensitive from resistant strains against $\mathrm{MQ}, \mathrm{QN}$ and $\mathrm{CQ}$. Although overall $\mathrm{IC}_{50}$ values obtained from the HRP-2 ELISA were slightly higher than those from the MSF method (Figure 2A), significant correlations between both methods were found in all drugs tested ( $r=0.73-0.99)$, except for DHA (Figure 3A). Analysis of pooled $\mathrm{IC}_{50} \mathrm{~S}$ of all drugs tested revealed that both methods were significantly correlated ( $r=0.97, p<0.0001$, Figure 3A). A Bland-Altman plot of $\mathrm{IC}_{50}$ values for all drugs is shown in Figure 4A, indicating a bias of $-0.07 \log$ units and limit of agreement between the two methods from -0.36 to 0.23 in log scale, further suggesting that results produced from both assays are comparable when testing in vitro reference strains.

\section{Immediate ex vivo drug susceptibility testing}

A total of 41 fresh $P$. falciparum isolates were analysed for IEV drug susceptibility using the HRP-2 and MSF assays in parallel. Success rate, defined as the proportion of samples in which it is possible to determine an $\mathrm{IC}_{50}$ value from a sigmoidal curve, appears to be dependent on baseline parasitaemia, as shown in Table 1. The HRP-2 ELISA demonstrated a high success rate (90\%), whereas the MSF method was successful at determining $\mathrm{IC}_{50} \mathrm{~S}$ in only $40 \%$ of samples. Of the total isolates tested, $\mathrm{IC}_{50}$ values were determined for 15 isolates using both assays. These 15 paired $\mathrm{IC}_{50}$ comparisons demonstrated consistently lower $\mathrm{IC}_{50} \mathrm{~s}$ obtained from the HRP-2 ELISA than those from the MSF method for MQ, QN, and CQ (Figure 2B). Significant correlations between $\mathrm{IC}_{50} \mathrm{~S}$ obtained from the two methods were found in all drugs tested, $r=0.56-0.84$, except for DHA and AS, and good correlation was found for pooled $\mathrm{IC}_{50} \mathrm{~S}$ against all drugs $(r=0.94, P<0.0001$, Figure $3 \mathrm{~B})$. A Bland-Altman plot of $\mathrm{IC}_{50} \mathrm{~s}$ for all drugs tested is shown in Figure 4B, indicating a bias of 0.12 and limit of agreement between two methods from -0.41 to 0.64 in log scale, indicating tight correlation between the two methods when evaluating field isolates.

\section{Reliability of HRP-2 and MSF assays in samples with low parasitaemia}

Two independent experiments were performed using W2 and D6 parasites to determine the lowest parasitaemia capable of producing sigmoidal concentration-response curves to determine $\mathrm{IC}_{50}$ values using the HRP-2 and MSF assays. Samples with a parasitaemia of 0.005, 0.01, $0.05,0.2$, and $0.5 \%$ were tested for in vitro drug susceptibility. For HRP-2 ELISA, a valid $\mathrm{IC}_{50}$ value was determined with minimal baseline parasitaemia as low as $0.005 \%$ or $0.05 \%$, depending on the rate of parasite growth during 72 hours' incubation. In contrast, the MSF assay requires an approximately one order of magnitude higher baseline parasitaemia range of $0.01-0.2 \%$, relative to direct comparison using HRP-2 ELISA in the same samples, to produce $\mathrm{IC}_{50}$ values.

Similarly, when evaluating IEV isolates, the MSF overall success rate (37\%) was lower than for HRP-2 ELISA (90\%). The MSF assay required $\geq 0.2 \%$ baseline parasitaemia for success, whereas the HRP-2 assay can produced results from isolates with parasitaemia $<0.2 \%$ (Table 1 ).

\section{Effect of \% parasitaemia on $I C_{50}$ values}

The effect of low baseline parasitaemia applied to the HRP-2 assay was evaluated because lower parasitaemia samples could produce an 'inoculum effect', a potential source for drug sensitivity test result variability resulting in lower $\mathrm{IC}_{50}$ values produced with lower baseline parasite density [24]. Low baseline parasitaemia for P. falciparum W2 and D6 reference strains of 0.005, 0.01, 0.05, 0.2, and $0.5 \%$ were tested in the HRP-2 assay in 2 independent experiments. This range of parasitaemia was chosen because it is representative of parasite densities encountered 


\section{A. $P$. falciparum reference clone results}

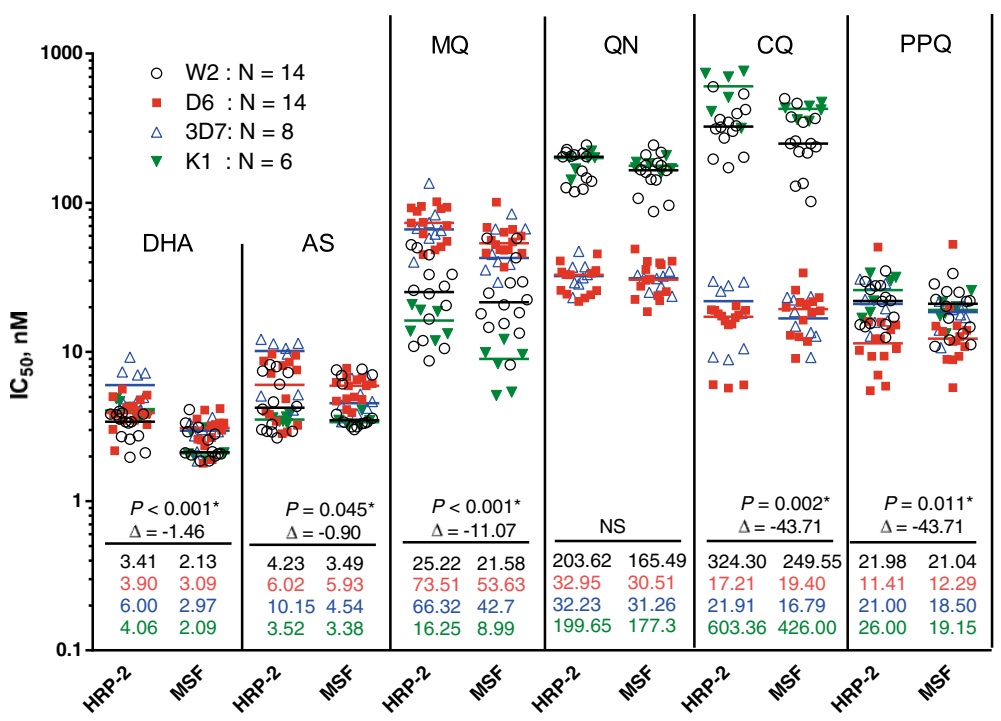

B. P. falciparum immediate ex vivo isolate results

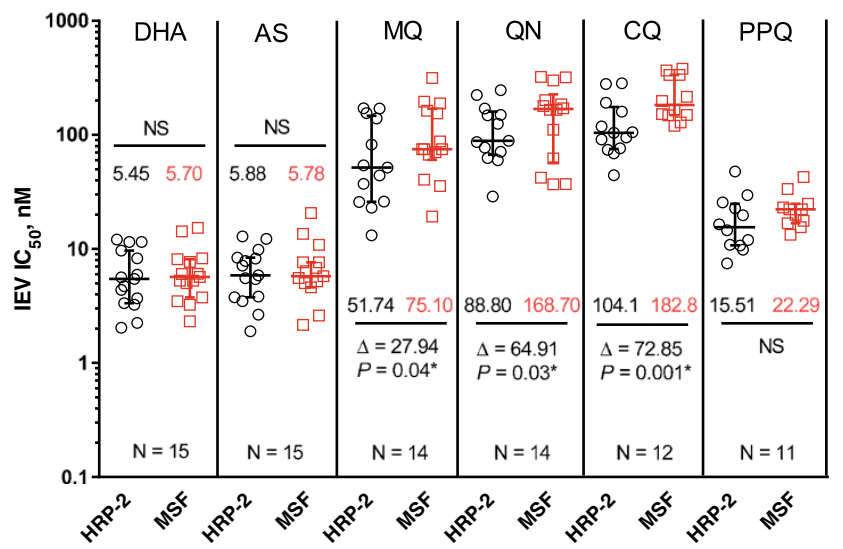

Figure 2 Direct comparison of $I C_{50}$ values obtained using the HRP-2 and MSF assays. (A) $I C_{50}$ s of reference laboratory clones (WBCs absent). Median IC $\mathrm{C}_{50}$ values for each drug against W2, D6, 3D7 and $\mathrm{K} 1$ strains are indicated using different symbols/colours $\left(n=\right.$ number of $I C_{50}$ values attained for each strain from 3-5 experiments). (B) IEV IC ${ }_{50}$ of field isolates (WBCs present). Of the total 41 IEV isolates evaluated in both the HRP-2 and MSF assays, $I_{50}$ values were obtained for both assays in 15 isolates. Median $I C_{50}$ values for field isolates obtained by HRP-2 and MSF assays are shown as black circles and red squares, respectively. Significant $p$-values of Wilcoxon matched pair tests comparing $I_{50} \mathrm{~S}$ from HRP-2 and MSF assays and their mean differences are designated with * symbol. NS signifies no statistical significance.

in Cambodian clinical isolates [9]. $\mathrm{IC}_{50}$ values were attained for all parasite densities tested for W2, whereas a parasitaemia $<0.05 \%$ did not yield $\mathrm{IC}_{50}$ results for D6 (Figure 5). The results suggest that for all drugs tested an inoculum effect was observed to a certain extent, yielding higher $\mathrm{IC}_{50}$ values with increasing parasitaemia. For instance, an inoculum effect was observed as statistically significant lower $\mathrm{IC}_{50}$ values for DHA and AS when evaluating samples with a low starting parasitaemia $(<0.2 \%)$ of P. falciparum W2 and D6 lines compared to higher parasitaemia samples (Figure 5A, 5B). For PPQ, significantly lower $\mathrm{IC}_{50}$ values were attained for a $\mathrm{W} 2$ baseline parasitaemia $<0.05 \%$ (Figure $5 \mathrm{D}$ ). For $\mathrm{CQ}$, an inoculum effect appears more obvious in the CQ-resistant W2 line relative to the CQ-sensitive D6 strain (Figure 5F). Likewise, a more noticeable inoculum effect is noted for MQ in the MQ-resistant D6 strain than in the MQ-susceptible W2 clone (Figure 5C).

\section{Discussion}

This is the first study to report the direct comparison of two field applicable methods, the HRP-2 and MSF assays, for evaluating drug resistance trends in immediate ex vivo isolates from Southeast Asia. Although several 


\section{A. P.falciparum reference clone results}

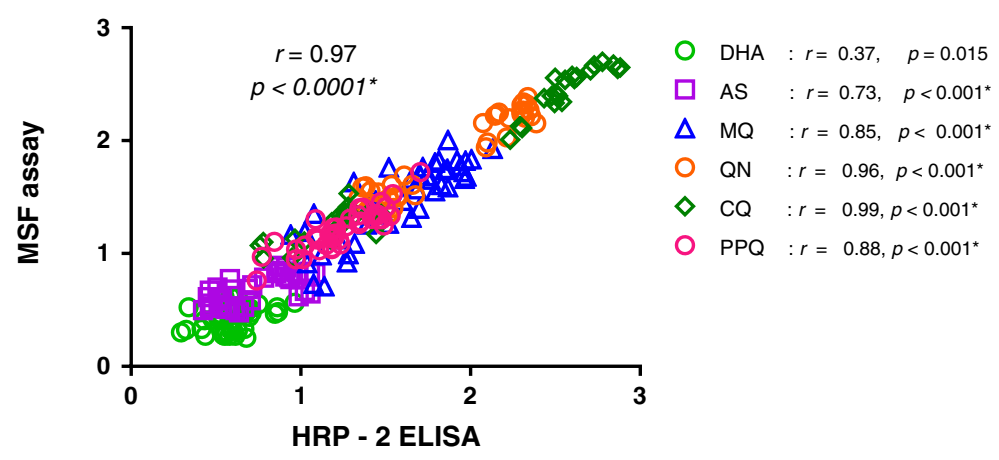

\section{B. P. falciparum immediate ex vivo isolate results}

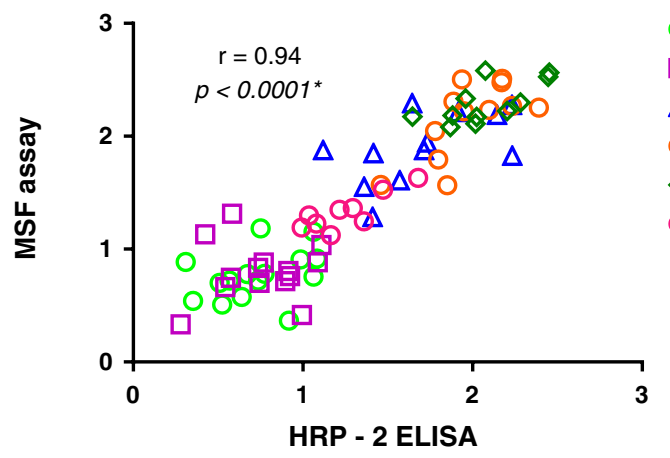

$\begin{array}{lll}\bigcirc & \text { DHA } & : r=0.34, p=0.208 \\ \square & \text { AS } & : r=0.07, p=0.788 \\ \Delta & \text { MQ } & : r=0.56, p=0.058^{\star} \\ \bigcirc & \text { QN } & : r=0.70, p=0.012^{\star} \\ \diamond & \text { CQ } & : r=0.67, p=0.023^{\star} \\ \bigcirc & \text { PPQ } & : r=0.84, p=0.005^{\star}\end{array}$

Figure 3 Correlations between HRP-2 and MSF assay $\log \left[\mathrm{IC}_{50}\right], \mathrm{nM}$, results for reference strains and field isolates. (A) $I_{50} S$ of reference laboratory clones (WBCs absent). (B) IEV IC $\mathrm{C}_{50}$ S of 15 field isolates (WBCs present). Scatter plots are shown for the correlation of log $\left[I C_{50} \mathrm{~S}\right]$ of all drugs tested by HRP-2 and MSF assays. $P$ value and $r$ from Pearson correlation are shown for all drugs together and each drug tested individually. Significant correlations are designated with * symbol.

studies demonstrate successful use of the MSF assay for evaluating P. falciparum isolates, all of these investigations used relatively high parasitaemia samples with mostly $\geq 0.2 \%$ parasitaemia from Africa $[6,17,25]$. However, results reported here using 41 fresh $P$. falciparum field isolates from Cambodia suggest that the HRP-2 ELISA offers superior sensitivity, compared to the MSF assay, in lower parasitaemia samples $(<0.2 \%)$ typical of malaria infections endemic to Southeast Asia. For instance, nearly half (49\%) of clinical isolates collected from prior drug resistance surveillance work in Cambodia in 2009-2012 were $<0.2 \%$ parasitaemia (AFRIMS data, $\mathrm{n}=209$ isolates). In contrast, only $22 \%$ of field isolates collected from Kenya in 2008-2009 were $<0.2 \%$ parasitaemia (data from Kenya Medical Research InstituteWalter Reed Project, Kisumu, $\mathrm{n}=292$ isolates). This discrepancy in the success of the two methods in clinical isolates appears to result from reduced sensitivity of the MSF assay in lower parasitaemia samples containing WBCs (Figure 1).

Good correlation of $\mathrm{IC}_{50}$ values for $\mathrm{MQ}, \mathrm{QN}, \mathrm{CQ}$, and PPQ was observed when comparing HRP-2 and MSF assay results, but it is unclear why poor correlation between methods was determined for the artemisinins (Figure 3). One possibility relates to assay reliability linked to mode and timing of drug action, since consistency of results for anti-malarial drug susceptibility assays depends on these drug-dependent variables [26]. Differences in methods for measuring parasite growth could possibly result in varying $\mathrm{IC}_{50}$ results. For instance, SYBR Green I fluorescence measures DNA replication and thus is less likely to produce results biased on parasite growth stage [27], whereas the HRP-2 protein is relatively stable and is actively secreted during parasite maturation stages [28]. However, lack of a true artemisinin-resistant reference strain hinders a meaningful comparison of methods. Unlike artemisinins, $P$. falciparum reference clones and field isolates are available that are resistant or sensitive to $\mathrm{MQ}, \mathrm{CQ}$ and $\mathrm{QN}$, allowing for robust comparisons of drug susceptibility assays [21]. In the absence of a standard artemisininresistant strain, further evaluation with more field isolates is needed to understand the sensitivities of the HRP-2 and MSF methods for differentiating artemisinin susceptibility from resistance. However, it is highly likely that in vitro assays will not detect resistance to fast-acting drugs, such as the artemisinins, and delayed parasite clearance 


\section{A. P.falciparum reference clone results}

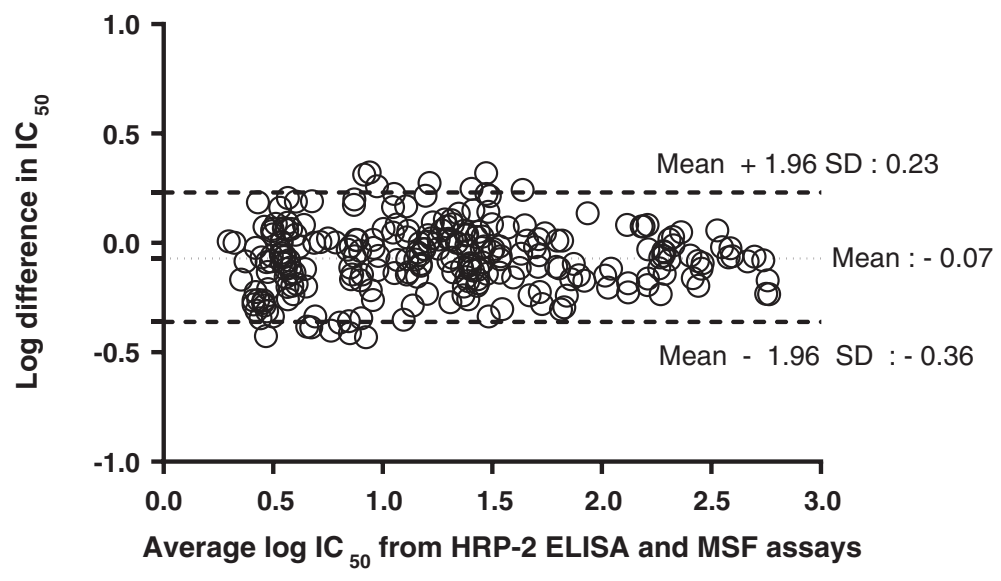

\section{B. $P$. falciparum immediate ex vivo isolate results}

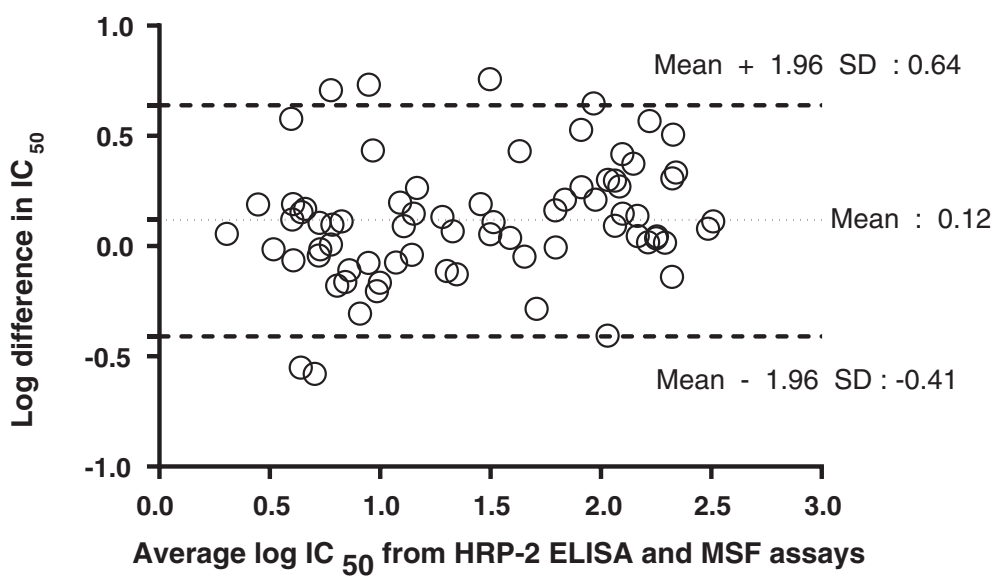

Figure 4 Bland-Altman plots of the difference in log $\mathrm{IC}_{50} \mathrm{~s}$ comparing HRP-2 and MSF assays. (A) Bland-Altman analysis results for reference laboratory clones (WBCs absent). (B) Results for 15 IEV isolates (WBCs present) in which $\mathrm{IC}_{50}$ values were obtained for both assays.

time and rate may continue to be the gold standard means for detecting artemisinin resistance $[29,30]$.

Besides considering mechanism of drug action, the effect of baseline parasitaemia is another variable to consider when interpreting drug sensitivity assay results

Table 1 Success rates for HRP-2 and MSF assays in fresh Plasmodium falciparum isolates of varying parasitaemia

\begin{tabular}{llll}
\hline \multirow{2}{*}{$\%$ parasitaemia } & \multicolumn{2}{l}{ Success case (\% success rate) } & \multirow{2}{*}{$\begin{array}{c}\text { Total } \\
\text { cases }\end{array}$} \\
\cline { 2 - 3 } & HRP-2 ELISA & MSF assay & \\
\hline$<0.02 \%$ & $1(50 \%)$ & $0(0 \%)$ & 2 \\
$0.02-<0.2 \%$ & $13(87 \%)$ & $0(0 \%)$ & 15 \\
$\geq 0.2 \%$ & $23(96 \%)$ & $15(63 \%)$ & 24 \\
Overall success & $37(90 \%)$ & $15(37 \%)$ & 41 \\
\hline
\end{tabular}

A total of 41 IEV Plasmodium falciparum isolates from Cambodia were evaluated in both the HRP- 2 and MSF assays to determine \% assay success rate. from clinical isolates. In the HRP-2 assay, a significant reduction in DHA and $\mathrm{AS} \mathrm{IC}_{50}$ values was observed when testing samples with a low starting parasitaemia $(<0.2 \%)$ of $P$. falciparum W2 and D6 lines relative to higher parasite densities (Figure 5). This finding has been noted previously in a study reporting higher artemisinin $\mathrm{IC}_{50}$ values, and thus an overestimation of artemisinin resistance, associated with an inoculum effect in Gambian field isolates [24]. Interestingly, for $C Q$, an inoculum effect was only noted in the CQ-sensitive D6 line, whereas baseline parasitaemia did not appear to alter HRP-2 assay results in the CQ-resistant W2 strain (Figure 5). A difference in the chloroquine cellular accumulation ratio between chloroquine-resistant and chloroquine-sensitive strains could explain these findings. In particular, kinetic studies suggest a faster rate of chloroquine accumulation in chloroquine-sensitive parasites and greater extent of drug 
A.

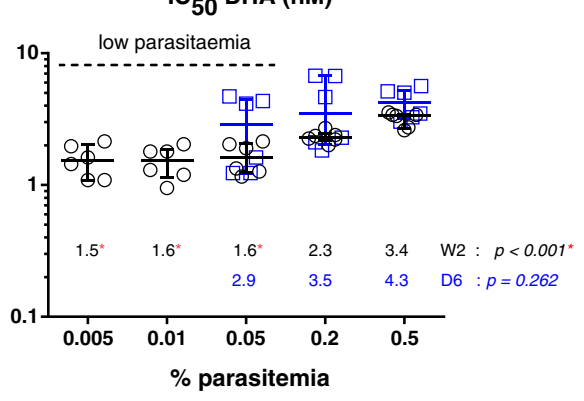

C.

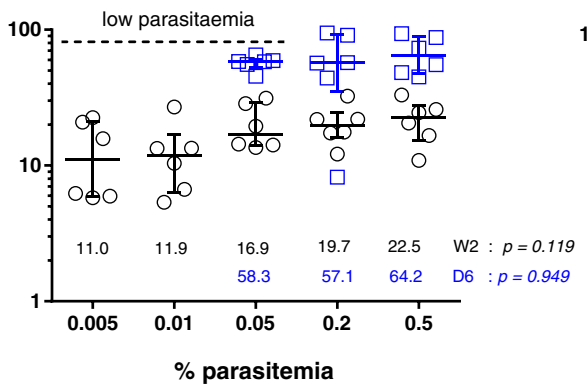

E.

$\mathrm{IC}_{50} \mathrm{QN}(\mathrm{nM})$

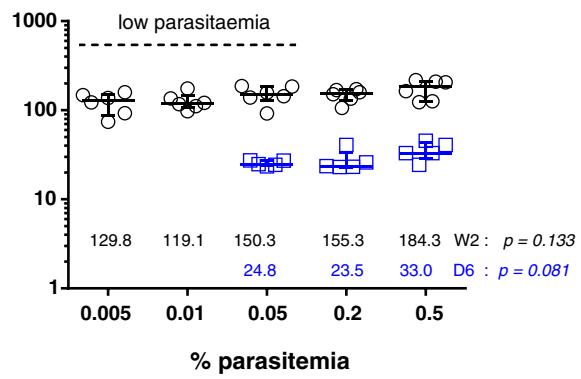

B.

IC ${ }_{50}$ AS (nM)

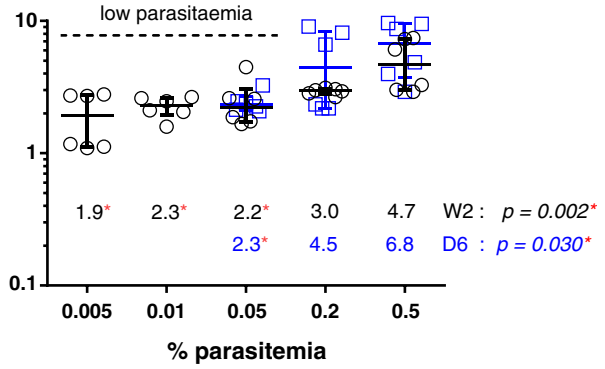

D.

$\mathrm{IC}_{50} \mathrm{PPQ}(\mathrm{nM})$

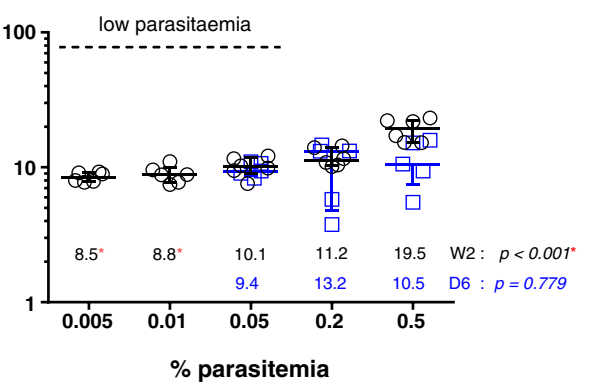

F.

$\mathrm{IC}_{50} \mathrm{CQ}(\mathrm{nM})$

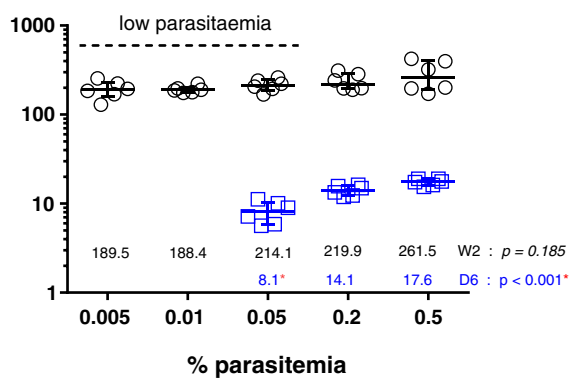

Figure 5 Effect of baseline parasitaemia on $I_{50}$ values for drugs tested by HRP-2 ELISA. (A) DHA, (B) AS, (C) MQ, (D) PPQ, (E) QN, and (F) CQ. Results presented are $I C_{50}$ values for drugs against W2 and D6 strains obtained from 2 independent experiments. The Kruskal-Wallis test was utilized to compare $I C_{50}$ values when testing a range of parasitaemias from 0.005 to $0.5 \%$ for W2 parasites and from 0.05 to $0.5 \%$ for D6 parasites. Red stars indicate significant $p$-values and reduced $I C_{50}$ values compared to those attained from a $0.5 \%$ parasitaemia baseline $(p<0.05$ by Dunn's Multiple comparison). Black circles and blue squares indicate $I_{50}$ values against W2 and D6, respectively. The dashed line in each graph indicates low baseline parasitaemia $(<0.2 \%)$.

being depleted from the cellular growth medium [31], which could result in greater concentrations of drug (i.e., higher $\mathrm{IC}_{50}$ values) required to kill chloroquine-sensitive strains with increases in inoculum size [32,33]. Overall, these results suggest that lower baseline parasitaemias reduce HRP-2 IC $_{50}$ values when evaluating highly susceptible, rather than drug-resistant, parasites.

Thus, it is possible that $\mathrm{IC}_{50}$ values could be lower for the artemisinins against susceptible clinical isolates of lower parasitaemias $(<0.2 \%)$. Indeed, in comparing HRP-2 assay $\mathrm{IC}_{50}$ results attained with parasitaemia $<0.2 \%$ versus $\geq 0.2 \%$ in a total of 350 IEV isolates from Cambodia collected in 2009-2012, significantly higher DHA and AS
$\mathrm{IC}_{50}$ values were observed in samples with $\geq 0.2 \%$ parasitaemia (median $\mathrm{IC}_{50}$ of 8.2 for DHA and 6.5 for AS) compared to those attained from isolates with $<0.2 \%$ parasitaemia (median $\mathrm{IC}_{50}$ of 6.2 for DHA and 4.1 for AS). However, interpretation of these results is complicated since isolates represent a mix of parasite populations, with potentially varying degrees of artemisinin susceptibility. To avoid potential confounding results, consistency in assay baseline parasitaemia was maintained to appropriately evaluate drug resistance surveillance results in field isolates [9].

The main conclusion of the present study is that HRP-2 ELISA is a more effective field-ready method, compared 
to the MSF assay, for analysing fresh clinical isolates of relatively low parasitaemia. However, as reported in this investigation and in previous reports [8,34], WBC depletion to decrease non-specific SYBR Green fluorescence I is expected to improve MSF assay sensitivity. Moreover, elimination of haemoglobin that also interferes with fluorescence readings was found to increase signal and assay sensitivity for low parasitaemia samples [34]. However, such additional sample processing steps to improve MSF assay performance in low parasitaemia isolates may ultimately be time consuming and labour intensive to justify practical use in the field. Since conducting this study, a modified version of the MSF assay [35] was developed involving a higher concentration of SYBR Green I dye and other modifications that could improve performance of the assay in field samples. Future efforts will investigate the effect of improving MSF assay performance with leucocyte depleted clinical isolates from Cambodia.

\section{Conclusions}

In the context of clinical samples not processed for WBC depletion, HRP-2 ELISA is superior to the MSF assay at evaluating fresh $P$. falciparum isolates with low parasitaemia $(<0.2 \%)$ typical of malaria endemic to Southeast Asia. Follow-on efforts are aimed at determining if performance of the MSF assay can be improved in fresh clinical isolates of low parasitaemia by removing WBCs to reduce nonspecific SYBR Green I fluorescence. Ultimately, field deployable malaria drug sensitivity assays must be convenient, requiring minimal sample preparation and relatively simple methods, and produce reliable results suitable for monitoring drug resistance specific to the malaria-endemic region of interest.

\section{Endnote}

The opinions and assertions contained herein are the private views of the authors and are not to be construed as official or as reflecting the views of the US Department of the Army. All human use research received the required ethical approvals from the appropriate authorities.

\section{Competing interests}

The authors declare that they have no competing interests.

\section{Authors' contributions}

SC designed the study, conducted experiments, analysed and interpreted data and wrote the manuscript. SDT participated in study design, results interpretation, manuscript preparation, project conception and management. KY, SS and PS conducted experiments, collected and analysed data. WR and JDJ contributed to results interpretation and manuscript preparation. DSW and DLS contributed to project conception and management. $\mathrm{CL}$ participated in design of the study, conducted experiments, collected data and contributed to project conception and management. CAL participated in design of the study, interpreted data and wrote the manuscript. All authors read and approved the final manuscript.

\section{Acknowledgements}

We are grateful to the AFRIMS and Cambodian clinical and laboratory field teams for conducting microscopy and their technical support. We thank Mr. William Ellis at the Walter Reed Army Institute of Research (WRAIR) for providing us with test drugs. We are appreciative of our colleagues at AFRIMS for their assistance: Dr. Delia Bethell for reviewing the paper, Ms. Somporn Krasaesub for advice on statistical analysis, and Ms. Panjaporn Chaichana for assistance with inoculum effect experiments. We thank Mr. Hoseah Akala, of the United States Army Kenya Medical Research Institute (KEMRI)-Walter Reed Project, Kisumu, for kindly providing data on the parasitaemia of $P$. falciparum isolates collected in Kenya. This work was funded by the Global Emerging Infections Surveillance (GEIS) Program, US Department of Defense.

\section{Author details}

'Department of Immunology and Medicine, Armed Forces Research Institute of Medical Sciences, Bangkok, Thailand. ${ }^{2}$ Department of Emerging Infectious Diseases Program, US Army Medical Research Unit-Kenya, Kenya Medical Research Institute-Walter Reed Project, Kisumu, Kenya.

Received: 2 April 2013 Accepted: 3 July 2013

Published: 12 July 2013

\section{References}

1. Rieckmann $\mathrm{KH}$ : Visual in-vitro test for determining the drug sensitivity of Plasmodium falciparum. Lancet 1982, 1:1333-1335.

2. Desjardins RE, Canfield CJ, Haynes JD, Chulay JD: Quantitative assessment of antimalarial activity in vitro by a semiautomated microdilution technique. Antimicrob Agents Chemother 1979, 16:710-718.

3. Noedl H, Attlmayr B, Wernsdorfer WH, Kollaritsch H, Miller RS: A histidine-rich protein 2-based malaria drug sensitivity assay for field use. AmJTrop Med Hyg 2004, 71:711-714.

4. Noedl H, Wernsdorfer WH, Miller RS, Wongsrichanalai C: Histidine-rich protein II: a novel approach to malaria drug sensitivity testing. Antimicrob Agents Chemother 2002, 46:1658-1664.

5. Johnson JD, Dennull RA, Gerena L, Lopez-Sanchez M, Roncal NE, Waters NC: Assessment and continued validation of the malaria SYBR green I-based fluorescence assay for use in malaria drug screening. Antimicrob Agents Chemother 2007, 51:1926-1933.

6. Akala HM, Eyase FL, Cheruiyot AC, Omondi AA, Ogutu BR, Waters NC, Johnson JD, Polhemus ME, Schnabel DC, Walsh DS: Antimalarial drug sensitivity profile of western Kenya Plasmodium falciparum field isolates determined by a SYBR green I in vitro assay and molecular analysis. AmJTrop Med Hyg 2011, 85:34-41.

7. Smilkstein M, Sriwilaijaroen N, Kelly JX, Wilairat P, Riscoe M: Simple and inexpensive fluorescence-based technique for high-throughput antimalarial drug screening. Antimicrob Agents Chemother 2004, 48:1803-1806.

8. Vossen MG, Pferschy S, Chiba P, Noedl H: The SYBR green I malaria drug sensitivity assay: performance in low parasitemia samples. AmJTrop Med Hyg 2010, 82:398-401.

9. Tyner SD, Lon C, Se Y, Bethell D, Socheat D, Noedl H, Sea D, Satimai W, Schaecher K, Rutvisuttinunt W, Fukuda MM, Chaorattanakawee S, Yingyuen K, Sundrakes S, Chaichana P, Saingam P, Buathong N, Sriwichai S, Chann S, Timmermans A, Saunders DL, Walsh DS: Ex vivo drug sensitivity profiles of plasmodium falciparum field isolates from Cambodia and Thailand, 2005 to 2010, determined by a histidinerich protein-2 assay. Malar J 2012, 11:198.

10. Bethell D, Se Y, Lon C, Tyner S, Saunders D, Sriwichai S, Darapiseth S, Teja-Isavadharm P, Khemawoot P, Schaecher K, Ruttvisutinunt W, Lin J, Kuntawungin W, Gosi P, Timmermans A, Smith B, Socheat D, Fukuda MM: Artesunate dose escalation for the treatment of uncomplicated malaria in a region of reported artemisinin resistance: a randomized clinical trial. PLoS One 2011, 6:e19283.

11. Noedl H, Bronnert J, Yingyuen K, Attlmayr B, Kollaritsch H, Fukuda M: Simple histidine-rich protein 2 double-site sandwich enzyme-linked immunosorbent assay for use in malaria drug sensitivity testing. Antimicrob Agents Chemother 2005, 49:3575-3577.

12. Noedl H, Se Y, Sriwichai S, Schaecher K, Teja-Isavadharm P, Smith B, Rutvisuttinunt W, Bethell D, Surasri S, Fukuda MM, Socheat D, Lon C: Artemisinin resistance in Cambodia: a clinical trial designed to address an emerging problem in Southeast Asia. Clin Infect Dis 2010, 51:e82-e89. 
13. Le Bras J, Deloron P, Ricour A, Andrieu B, Savel J, Coulaud JP: Plasmodium falciparum: drug sensitivity in vitro of isolates before and after adaptation to continuous culture. Exp Parasitol 1983, 56:9-14.

14. Nsobya SL, Kiggundu M, Joloba M, Dorsey G, Rosenthal PJ: Complexity of Plasmodium falciparum clinical samples from uganda during short-term culture. J Infect Dis 2008, 198:1554-1557.

15. Pelleau S, Bertaux L, Briolant S, Ferdig MT, Sinou V, Pradines B, Parzy D, Jambou R: Differential association of Plasmodium falciparum $\mathrm{Na}+\mathrm{H}+$ exchanger polymorphism and quinine responses in field- and cultureadapted isolates of Plasmodium falciparum. Antimicrob Agents Chemother 2011, 55:5834-5841

16. Bacon DJ, Latour C, Lucas C, Colina O, Ringwald P, Picot S: Comparison of a SYBR green I-based assay with a histidine-rich protein II enzyme-linked immunosorbent assay for in vitro antimalarial drug efficacy testing and application to clinical isolates. Antimicrob Agents Chemother 2007, 51:1172-1178

17. Rason MA, Randriantsoa T, Andrianantenaina H, Ratsimbasoa A, Menard D: Performance and reliability of the SYBR green I based assay for the routine monitoring of susceptibility of Plasmodium falciparum clinical isolates. Trans R Soc Trop Med Hyg 2008, 102:346-351.

18. Trager W, Jensen JB: Human malaria parasites in continuous culture. Science 1976, 193:673-675.

19. Chaijaroenkul W, Bangchang KN, Mungthin M, Ward SA: In vitro antimalarial drug susceptibility in Thai border areas from 1998-2003. Malar J 2005, 4:37.

20. Lambros C, Vanderberg JP: Synchronization of Plasmodium falciparum erythrocytic stages in culture. J Parasitol 1979, 65:418-420.

21. Rutvisuttinunt W, Chaorattanakawee $S$, Tyner SD, Teja-Isavadharm P, Se $Y$, Yingyuen K, Chaichana P, Bethell D, Walsh DS, Lon C, Fukuda M, Socheat D, Noedl H, Schaecher K, Saunders DL: Optimizing the HRP-2 in vitro malaria drug susceptibility assay using a reference clone to improve comparisons of Plasmodium falciparum field isolates. Malar J 2012, 11:325.

22. Le Nagard H, Vincent C, Mentre F, Le Bras J: Online analysis of in vitro resistance to antimalarial drugs through nonlinear regression. Comput Methods Programs Biomed 2011, 104:10-18.

23. Bland JM, Altman DG: Comparing methods of measurement: why plotting difference against standard method is misleading. Lancet 1995, 346:1085-1087.

24. Duraisingh MT, Jones $P$, Sambou I, von Seidlein $L$, Pinder M, Warhurst DC: Inoculum effect leads to overestimation of in vitro resistance for artemisinin derivatives and standard antimalarials: a Gambian field study. Parasitology 1999, 119(Pt 5):435-440.

25. Basco LK: Molecular epidemiology of malaria in Cameroon. XX. experimental studies on various factors of in vitro drug sensitivity assays using fresh isolates of Plasmodium falciparum. AmJTrop Med Hyg 2004, 70:474-480.

26. Wein S, Maynadier M, Tran Van Ba C, Cerdan R, Peyrottes S, Fraisse L, Vial H: Reliability of antimalarial sensitivity tests depends on drug mechanisms of action. J Clin Microbiol 2010, 48:1651-1660.

27. Smeijsters LJ, Zijlstra NM, Franssen FF, Overdulve JP: Simple, fast, and accurate fluorometric method to determine drug susceptibility of Plasmodium falciparum in 24-well suspension cultures. Antimicrob Agents Chemother 1996, 40:835-838.

28. Desakorn V, Silamut K, Angus B, Sahassananda D, Chotivanich K, Suntharasamai P, Simpson J, White NJ: Semi-quantitative measurement of Plasmodium falciparum antigen PfHRP2 in blood and plasma. Trans $R$ SoC Trop Med Hyg 1997, 91:479-483.

29. Noedl H, Se Y, Schaecher K, Smith BL, Socheat D, Fukuda MM: Evidence of artemisinin-resistant malaria in western Cambodia. N Engl J Med 2008, 359:2619-2620.

30. O'Brien C, Henrich PP, Passi N, Fidock DA: Recent clinical and molecular insights into emerging artemisinin resistance in Plasmodium falciparum. Curr Opin Infect Dis 2011, 24:570-577.

31. Geary TG, Divo AD, Jensen JB, Zangwill M, Ginsburg H: Kinetic modelling of the response of Plasmodium falciparum to chloroquine and its experimental testing in vitro. implications for mechanism of action of and resistance to the drug. Biochem Pharmacol 1990, 40:685-691.

32. Hawley SR, Bray PG, Mungthin M, Atkinson JD, O'Neill PM, Ward SA: Relationship between antimalarial drug activity, accumulation, and inhibition of heme polymerization in Plasmodium falciparum in vitro. Antimicrob Agents Chemother 1998, 42:682-686.

33. Bray $P G$, Mungthin M, Ridley RG, Ward SA: Access to hematin: the basis of chloroquine resistance. Mol Pharmacol 1998, 54:170-179.

34. Moneriz C, Marin-Garcia P, Bautista JM, Diez A, Puyet A: Haemoglobin interference and increased sensitivity of fluorimetric assays for quantification of low-parasitaemia plasmodium infected erythrocytes. Malar J 2009, 8:279.

35. P. falciparum drug sensitivity assay using SYBR ${ }^{\circledast}$ Green I Assay Technique WWARN Procedure: In vitro Module. WWARN; 2011.

doi:10.1186/1475-2875-12-239

Cite this article as: Chaorattanakawee et al:: Direct comparison of the histidine-rich protein-2 enzyme-linked immunosorbent assay (HRP-2 ELISA) and malaria SYBR green I fluorescence (MSF) drug sensitivity tests in Plasmodium falciparum reference clones and fresh ex vivo field isolates from Cambodia. Malaria Journal 2013 12:239.

\section{Submit your next manuscript to BioMed Central and take full advantage of:}

- Convenient online submission

- Thorough peer review

- No space constraints or color figure charges

- Immediate publication on acceptance

- Inclusion in PubMed, CAS, Scopus and Google Scholar

- Research which is freely available for redistribution 